Schmerz 2019 $\cdot 33: 261-262$

https://doi.org/10.1007/s00482-019-0377-z

(c) Springer Medizin Verlag GmbH, ein Teil von Springer Nature 2019

CrossMark

\author{
P. Herrmann ${ }^{1} \cdot$ R. Griebenow ${ }^{2}$ \\ ${ }^{1}$ Springer Medizin Verlag, Heidelberg, Deutschland \\ ${ }^{2}$ Ärztekammer Nordrhein, Düsseldorf, Deutschland
}

\title{
Erklärung zu finanziellen und nichtfinanziellen Interessen in CME-Fortbildungen von Springer Medizin
}

\section{Umsetzung der „Richtlinie der Ärztekammer Nordrhein zur Fortbildungsordnung"}

Liebe Kolleginnen, liebe Kollegen,

der hohe Qualitätsanspruch von CMEBeiträgen zeigt sich nicht nur an der wissenschaftlichen Exzellenz, der guten Passung für die ärztliche Praxis, sondern auch in der Unabhängigkeit von Herausgebern, Autoren und Redaktion. Daher hat der Verlag mit Beginn der Fortbildungspflicht in 2004 begonnen, systematisch und kontinuierlich die Regeln zur Sicherung der Transparenz für Herausgeber, Autoren, Leser und Teilnehmer einzuführen und zu verbessern. Insbesondere die Konformität mit der Richtlinie der Ärztekammer sowie die Regelung zu den möglichen Interessenkonflikten sind seither ein wesentlicher Bestandteil einer jeden zertifizierten Fortbildung.

Einige dieser Regeln werden nun nach der aktuellen Richtlinie der Ärztekammer Nordrhein angepasst. Dies haben wir von Seiten Springer Medizin Verlag in enger Abstimmung mit der Ärztekammer Nordrhein vorbereitet. Wir stellen Ihnen für Ihre Zeitschrift die wichtigsten Änderungen vor.

Die Erklärung von Interessen (häufig auch als „Interessenkonflikte“ bezeich-

Dieser Beitrag erscheint in allen Fachzeitschriften von Springer Medizin, deren CME-Fortbildungen von der Ärztekammer Nordrhein zertifiziert werden.

Erstveröffentlichung in DerUrologe(2019)58:1-2. https://doi.org/10.1007/s00120-018-0841-x. net) ist integraler Bestandteil unabhängiger, von den deutschen Ärztekammern zertifizierter Fortbildungsmaßnahmen. Die (Muster-)Fortbildungsordnung (FBO, abrufbar unter: www. bundesaerztekammer.de/fileadmin/ user_upload/downloads/_Muster_Fortbildungsordnung_29052013.pdf) legt hierzu fest:

S 8: „... Interessenkonflikte des Veranstalters, der wissenschaftlichen Leitung und der Referentinnen und Referenten müssen gegenüber den Teilnehmerinnen und Teilnehmern an der Fortbildungsmaßnahme offengelegt werden."

Ziel dieser Offenlegung soll laut den „Empfehlungen der Bundesärztekammer zur ärztlichen Fortbildung“ (abrufbar unter: www.bundesaerztekammer. de/fileadmin/user_upload/downloads/ pdf-Ordner/Fortbildung/EmpfFortb_ 20150424.pdf) sein, „dass der Teilnehmer sowie die anerkennende Ärztekammer die Möglichkeit erhalten, sich eine Meinung über die Interessenlage eines Veranstalters/Referenten/ wissenschaftlichen Leiters zu bilden. Im Vordergrund steht die Information, nicht die Ausgrenzung aufgrund von Verbindungen zur Industrie oder anderen Gruppierungen.“

Daraus folgt, dass Umfang und Art der Offenlegung geeignet sein müssen, eine solche Meinungsbildung zu ermöglichen.
Um eine möglichst hohe Standardisierung in den einzelnen Kammern zu erreichen, legen die genannten Empfehlungen weiterhin fest:

„Für die Offenlegung von potentiellen Interessenkonflikten als Selbstauskunft stellt die Ärztekammer einen Fragebogen zur Verfügung. "Für von der Ärztekammer Nordrhein anerkannte Fortbildungsmaßnahmen ist dieser Fragebogen abrufbar unter: https://www.aekno. de/downloads/aekno/erklaerung-finanzinteressenkonflikt-2018-11.pdf

Die in diesem Fragebogen geforderten Angaben sind als Mindestangaben zu verstehen, die vom „Veranstalter" (im Fall dieser Zeitschrift vom Springer Medizin Verlag) nach Art und Umfang in dem Maße anzupassen bzw. zu erweitern sind, wie der Verlag es für ein zielführendes Management von Interessenkonflikten für notwendig erachtet.

Das für Springer Medizin Verlag geltende Formular finden Sie bei den Autorenrichtlinien der Zeitschrift auf www.springer.com

Darüber hinaus sei darauf verwiesen, dass seitens der Ärztekammer Nordrhein bezüglich Interessenkonflikten nur zwei Ausschlussgründe für die Zertifizierung eines CME-Beitrags in dieser Zeitschrift definiert sind:

- eine an Planung und Durchführung einer Fortbildungsmaßnahme be- 
teiligte Person legt keine Erklärung $\mathrm{zu}$ den eigenen Interessen vor. Um eine solche Situation generell zu vermeiden, legt die Richtlinie zur Fortbildungsordnung fest, dass die Erklärungen zu finanziellen und nichtfinanziellen Interessen zum Zeitpunkt der Antragstellung vorliegen müssen (d.h. im Falle dieser Zeitschrift idealerweise im Prozess der Manuskripterstellung mit erhoben werden).

- zur Wahrung der nach FBO und SGB V (\$\$95d, 136) geforderten Freiheit der Inhalte von Fortbildung von wirtschaftlichen Interessen dürfen nach den „Empfehlungen“ Mitarbeiter eines Sponsors grundsätzlich nicht als Referenten, Kursleiter oder Autoren bei einer Fortbildungsmaßnahme mitwirken.

Dieser Punkt kommt für die Zeitschrift nicht zum Tragen, denn unter der Rubrik „Zertifizierte Fortbildung“ werden grundsätzlich keine gesponserten Inhalte publiziert.

Davon unbenommen und unabhängig von der Zertifizierung durch die Kammer liegt die Bewertung bzgl. inhaltlicher Qualität bzw. Begutachtung und Annahme eines Beitrags vollumfänglich und unverändert bei den Herausgebern der Zeitschrift.

\section{Fazit}

Die Vorgaben der Ärztekammer werden zukünftig in dieser Zeitschrift so umgesetzt, dass die Leser und Teilnehmer

- die entsprechenden Erklärungen der Herausgeber (entsprechend der „wissenschaftlichen Leitung“ im Sinne der Richtlinie) online am zertifizierten Kurs einsehen können unter www.springermedizin.de/cme,

- die Angaben der Autoren zu den finanziellen und nichtfinanziellen Interessen wie bisher am Ende eines jeden CME-Beitrags und online am zertifizierten Kurs einsehen können.

Dabei werden die Angaben zu den finanziellen Interessen der Autoren für alle Fragen, die mit ,ja“ beantwortet wurden, angegeben, während die nichtfinanziel- len Interessen in vollem Umfang aufgelistet werden.

Wir möchten hiermit einen Beitrag leisten $\mathrm{zu}$ Transparenz und Meinungsbildung. Dabei danken wir den Herausgebern und Autoren für ihre Bereitschaft mitzuwirken.

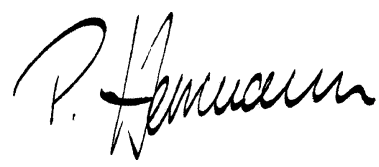

Dr. med. Paul Herrmann

Director Journals \& ePublishing, Springer Medizin Verlag

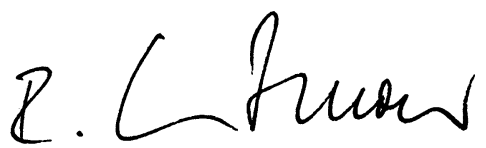

Prof. Dr. med. Reinhard Griebenow Vorsitzender Fortbildungsausschuss der Ärztekammer Nordrhein

\section{Korrespondenzadresse}

\section{Dr. med. P. Herrmann}

Springer Medizin Verlag

Tiergartenstraße 17, 69121 Heidelberg,

Deutschland

paul.herrmann@springernature.com

Interessenkonflikt. P. Herrmann gibt an, dass kein finanzieller Interessenkonflikt besteht. Nichtfinanzielle Interessen: P. Herrmann ist als Director Journals \& ePublishing beim Springer Medizin Verlag angestellt, der hier als Fortbildungsanbieter auftritt. Darüber hinaus ist P. Herrmann Mitglied im Berufsverband Deutscher Internisten, der Deutschen Schmerzgesellschaft und der Deutschen Gesellschaft für Innere Medizin, deren Sprecher der Korporativen Mitglieder er ist. R. Griebenow gibt an, dass kein finanzieller Interessenkonflikt besteht. Nichtfinanzielle Interessen: Niedergelassener Kardiologe, Praxis Rheingalerie Köln (2017-)|Oberarzt, Klinik für Kardiologie, Angiologie und Diabetologie, Kliniken der Stadt Köln, KölnMerheim (-2017)|Vorsitzender des Vorstands, European Cardiology Section Foundation|Vorsitzender des Advisory Committee, European Board for Accreditation in Cardiology|Stellvertr. Vorsitzender, Continuing Medical Education-European Accreditors (CME-EA)|Mitglied des Vorstands, Ärztekammer Nordrhein (ÄKNo),|Vorsitzender, Fortbildungsausschuss der ÄKNo|Vorsitzender, Ausschuss „Ausbildung zum Arzt, Hochschulen und Hochschulen und medizinischen Fakultäten" der ÄKNo|Mitglied, Ausschuss „Ärztliche Aus-, Weiter- und Fortbildung" der BÄK|Mitglied, Ständige Konferenz „Ärztliche Fortbildung" der BÄK|Vorsitzender, Marburger Bund Bezirk Köln (-2018)|Mitglied, Arbeitskreis Fort-und Weiterbildungspolitik, Marburger Bund|Mitglied, Weiter- und Fortbildungsakademie "Kardiologie" der Deutschen
Gesellschaft für Kardiologie (DGK)|Mitglied, Editorial Board, Journal of European CME (JECME)|Mitglied, Berufsverband Deutscher Internisten (BDI)|Past-President, UEMS Cardiology Section. 